\title{
Parameterization of the Construction Promoter's Responsibilities throughout the project's life cycle
}

Santiago Díez Torrijos

1ot Project Management,

Sofia Real Time Data

s.diez@1ot.es

Ricardo Ayala López

1ot Project Management,

Sofia Real Time Data

r.ayala@10t.es
Antonio Ramón Albert

1ot Project Management,

Sofia Real Time Data

a.ramon@10t.es

José Agustín Lillo Arnalte

1ot Project Management,

Sofia Real Time Data

a.lillo@10t.es
Benjamín Benlloch Miralles

1ot Project Management,

Sofia Real Time Data

b.benlloch@10t.es

Luis Tena Gil

Sofia Real Time Data

l.tena@sofia-rtd.com
DOI 10.5592/otmcj.2015.1.7

Tehnical paper

\section{Keywords}

Transparency, Project Portfolio Management, Construction Promoter, Parameterization, Decision Making Process, Real-time, Business Intelligence
EUROPE, AND ESPECIALLY SPAIN HAS BEEN SUFFERING AN ONGOING ECONOMIC, SOCIAL AND POLITICAL CRISIS. THE CONSTRUCTION INDUSTRY SECTOR HAS BEEN ONE OF THE MOST AFFECTED.

Project Management professionals in the construction industry are obliged to make a critical analysis of their results and methodology applications used in recent years.

In this paper a methodology has been developed for parameterization and monitoring of projects, portfolios and programs from both the public or private Construction Promoter's point of view -the real engine of the project-. This methodology analyses their responsibilities and those of the stakeholders throughout the project's lifecycle, and allows the Promoter to make decisions based on accurate, realistic and factual knowledge.

The project's full data is completely accessible by the Promoter. Thus the Promoter is able to develop a Project Portfolio Management and Business Intelligence.

The Next step in the methodology evolution is transferring it to an ICT tool, for a promoter's systematic application.

Project management will assist in providing a social improvement, this method is associated with current social demands at all levels and sectors -Transparency- thus allowing realtime monitoring of the building project's status throughout all stages of the project's life cycle. 


\section{INTRODUCTION}

The Construction Promoter -is the organization, specialized either within or outside the construction industrybeing its mission the development of construction projects, portfolios or programs with two final goals, operating the real estate asset for specific activities and sales procesess.

Seven phases can be identified in the develoment of any project, these are: Concept, Technical Definition, Contract, Licenses, Construction, Operating and Deactivation. For the Promoter, the construction phase is the most intensive, in terms of production, but not the most important in terms of responsabilities. Analysing the percentage represented for each phase within the Project's life cycle, assuming such a Project life cycle has 50 years (see figure $n-1$ ), it`s possible to observe phases associated within the construction process (from Technical Definition to Construction), assuming no more than $3-4 \%$ of the total time.

During periods of economic instability, as currently seen in Europe, specifically in Spain, where real estate assets are subject to sales processes (due diligence) or modifications -change of use, reforms or renovations- created by market forces. It is then that an appropiate project management's methodology from the initial stages of the project, determines the real success of the majority of these operations.

The organizational structure of the Promoter is comprised of a number of departments, that interact with the real estate assets throughout the entire lifecycle. Those departments across the organization as defined by the Promoter, and are directly involved in the decision making process which determines the projects success. Those departments include: Expansion, Financial, Legal, Operations, Maintenance, Safety, Development, Information Systems, Administration, and Communication.

Proceeding onwards in identifying all the Construction Promoter's responsibilities during the Project's seven phases, as mentioned before. Recognizing all parties involved within building projects in Spain (stakeholders), and how they interact and progress with the Promoter's responsibilities. Finally consideration for the parameterization's methodology of

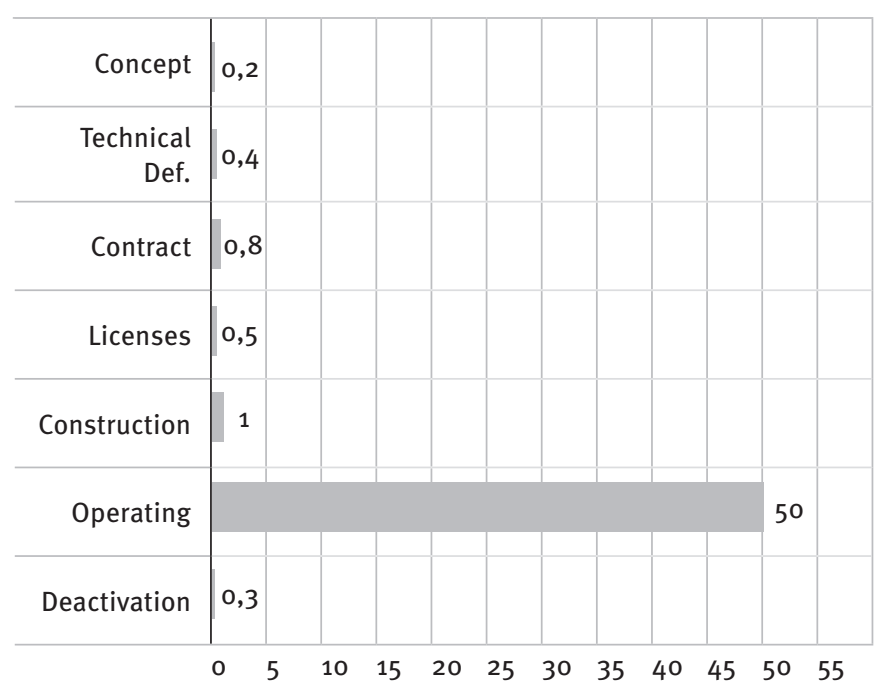

Figure 1. Duration of each phase for a project's life-cycle of 50 years

these responsibilities, that allows data collection and continuous measurement as a staple for the decisionmaking process.

All the figures shown are related to real projects described in heading number 5 , Practice Case.

\section{Responsibilities}

The Promoter's success of a building project must assumes achieving specific objectives, and project management is the process of control for the achievement of the project's objectives by application of a set of tools and techniques (López Paredes et al, 2013).

The Promoter has defined goals relative to each area of responsibility, the project's success will be measured by the Promoter's ability in fulfilling their responsibilities.

In the construction industry, the Promoter has ten responsibilities throughout the project's life cycle, portfolio or program (Díez Torrijos, 2015). These responsibilities are:
1. Finances
2. Planning
3. Production
4. Legal
5. Quality
6. Sustainability
7. Health \& Safety
8. Operating / Sales
9. Insurance
10. Maintenance

For each area of responsibility, this methodology defines what criteria can be considered in determining the fulfilment and how it can be evaluated. In this latter process - how can it be evaluated?- a change of approach is presented. In this paper it's proposed for the parameterization of all areas of responsibility a methodology for an easy data collection and simple measurement, and fundamentally, a continuous and full coverage comparison between the actual reality and the 

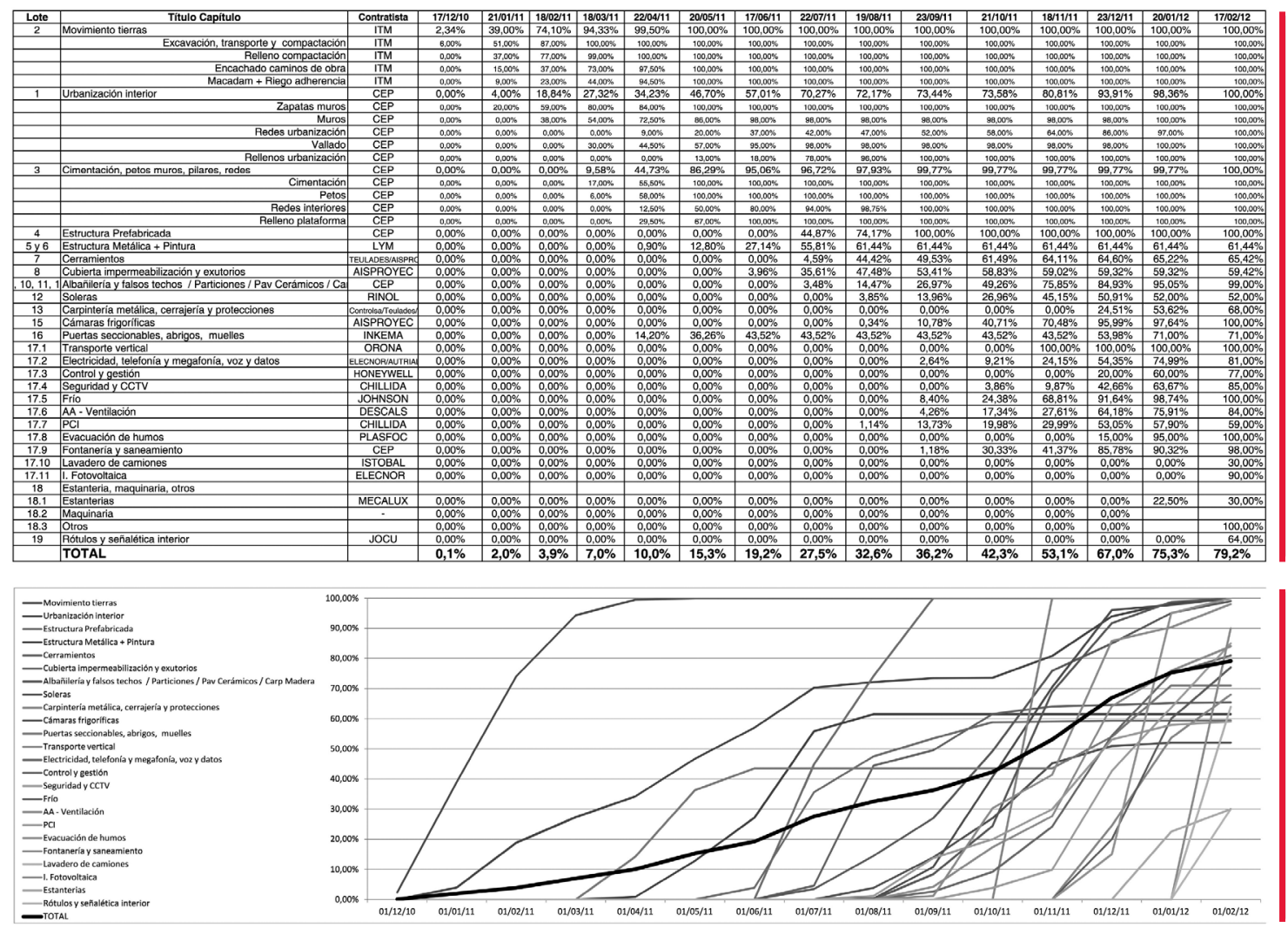

Figure 2. Cumulative production by chapter - Practice Case: Food logistic warehouse

theoretical data objectives of the project. Continuous comparison refers to the permanent comparison during each Project phase, within the active areas of responsibility. Full coverage refers to the comparison of all areas active simultaneously.

Parameterization methodology prioritizes the criteria at different levels depending on its relevance to the Promoter, offering a full Project insight. The methodology allows full information access for all areas of responsibility to include all the differing hierarchy levels information requirements.

Making a brief analysis for each area of responsibility, the primary criteria used within real estate projects, are related to:

Finance. Primary level: feasibility, cost and financial planning, profitability, financial parameters and invoicing. At other levels access to specific information by chapters and subchapters.
- Planning. Primary level: general planning, planning phases, and milestones. At other levels access to specific information by chapters, subchapters and tasks.

> Production. Primary level: general production of the Project. At other levels access to specific information by deliverable or work unit. e.g. figure 2, Chapters Construction Production from a real project.

$\checkmark$ Legal. Primary level: licenses, permits, supplies, agreements, contracts and settlements. At other levels access to detailed information for each relevant task or phase.

Q Quality. Primary level: quality plan. At other levels access to detailed information on chapters and subchapters. e.g. figure 3, Quality plan criteria from a real project.

> Sustainability. Primary level: under construction goals, objectives operating phase. At other levels access to detailed information on chapter and subchapter. e.g. figure 4, Sustainability Targets from a real project.

- Health and safety. Primary level: risks within the construction process. At other levels access to detailed information on chapters and subchapters. e.g. figure 5, Health and Safety criteria from a real project.

- Operations / sales. Primary level: feasibility cost and financial planning, profitability, financial parameters, invoicing, due diligence. At other levels access to detailed information on chapters and subchapters.

- Risk Management / Insurance. Primary level: project risks at each stage, and mandatory insurance coverage. At other levels access to detailed information on chapters and subchapters.

Maintenance. Primary level: preventive maintenance, corrective maintenance. At other levels access to detailed information on chapters 


\begin{tabular}{|c|c|c|c|c|c|}
\hline \multicolumn{6}{|c|}{ TABLA DEL SEGUIMIENTO DE EJECUCIÓN DE ENSAYOS } \\
\hline \multirow{3}{*}{ LOTE } & \multirow{2}{*}{\multicolumn{3}{|c|}{ A ORIGEN }} & \multirow{2}{*}{\multicolumn{2}{|c|}{$\frac{\text { SEMANA(24) }}{15 / 06-19 / 06 / 15}$}} \\
\hline & & & & & \\
\hline & $\begin{array}{l}\text { NÚMERO ENSAYOS } \\
\text { PREVISTOS }\end{array}$ & EJECUTADOS & DESFAVORABLES & EJEC. & DESF. \\
\hline $\begin{array}{c}1 \text { URBANIZACIÓN, CERRAMIENTO Y REDES } \\
\text { EXTERIORES }\end{array}$ & 147,00 & 5,00 & 0,00 & 0,00 & 0,00 \\
\hline $\begin{array}{l}4 \text { CIMENTACIONES, ENANOS, PETOS, } \\
\text { MURETES, FOSOS Y REDES ENTERRADAS }\end{array}$ & 251,20 & 243,48 & 22,56 & 18,00 & 0,00 \\
\hline $\begin{array}{l}5 \text { ESTRUCTURA METÁLICA Y PROTECCIÓN } \\
\text { IGNÍFUGA }\end{array}$ & 73,00 & 64,16 & 2,00 & 0,80 & 0,00 \\
\hline 6 ESTRUCTURA DE HORMIGÓN Y FORJADOS & 53,00 & 53,00 & 0,00 & 0,00 & 0,00 \\
\hline 7 CUBIERTA, CANALONES Y LUCERNARIOS & 18,00 & 6,00 & 0,00 & 3,00 & 0,00 \\
\hline 9 FACHADAS & 10,00 & 0,00 & 0,00 & 0,00 & 0,00 \\
\hline 10 SOLERAS DE HORMIGÓN Y RESINAS & 129,00 & 0,00 & 0,00 & 0,00 & 0,00 \\
\hline $\begin{array}{l}11 \text { ALBAÑILERÍA, REVESTIMIENTOS Y } \\
\text { ACABADOS }\end{array}$ & 33,00 & 18,00 & 0,00 & 12,00 & 0,00 \\
\hline $\begin{array}{c}18 \text { EVACUACIÓN PLUVIALES SISTEMAS DE } \\
\text { SUCCIÓN }\end{array}$ & 2,00 & 0,00 & 0,00 & 0,00 & 0,00 \\
\hline 20 CAMARAS FRIGORÍFICAS & 10,00 & 0,00 & 0,00 & 0,00 & 0,00 \\
\hline 21 FRÍO INDUSTRIAL & 236,00 & 0,00 & 0,00 & 0,00 & 0,00 \\
\hline 22 INSTALACIÓN ELECTRICIDAD & 285,00 & 0,00 & 0,00 & 0,00 & 0,00 \\
\hline $\begin{array}{l}23 \text { INSTALACIÓN PROTECCIÓN CONTRA } \\
\text { INCENDIOS }\end{array}$ & 29,40 & 0,00 & 0,00 & 0,00 & 0,00 \\
\hline 24 INSTALACIÓN AIRE ACONDICIONADO & 66,00 & 0,00 & 0,00 & 0,00 & 0,00 \\
\hline TOTAL & $1.342,60$ & 389,64 & 24,56 & 33,80 & 0,00 \\
\hline PORCENTA & E INCIDENCIAS SEMA & & & & \\
\hline
\end{tabular}

\begin{tabular}{l|c}
\hline SEGUIMIENTO PLAN DE CALIDAD (\% ENSAYOS EJECUTADOS A ORIGEN) & $29,02 \%$ \\
MEDIDA DE LA CALIDAD DE LA OBRA (\% ENSAYOS DESFAVORABLES A ORIGEN) & $6,30 \%$ \\
MEDIDA DE LA CALIDAD DE LA OBRA (\% ENSAYOS DESFAVORABLES DE LA ÚLTIMA SEMANA) & $0,00 \%$
\end{tabular}

* La totalidad de los ensayos desfavorables son vueltos a ensayar hasta obtener un resultado favorable

*Se han incluido los ensayos extras no previstos en presupuesto inicial

*El número de ensayos previstos variará en caso de tener que incluir ensayos extras no previstos en presupuesto inicial

*Para determinaciones de densidad de un suelo, temperatura del asfalto y espesor de pintura, 25 ensayos computan como 1 unidad en el presente informe

Weekly average test result by construction subchapter 


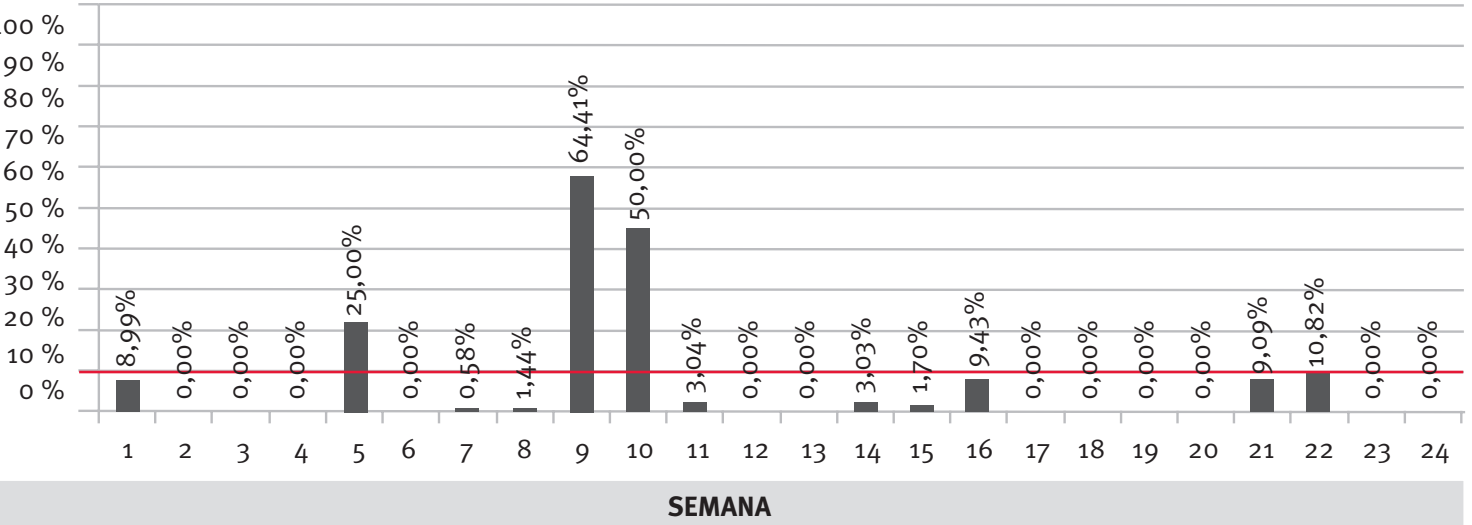

*La línea roja indica el límite máximo deseado de ensayos desfavorables (10\% de los ensayos semanales realizados).

Figure 3. Medida de la calidad de la obra

\begin{tabular}{|c|c|c|c|c|}
\hline \multicolumn{5}{|c|}{ TARGET - CONSTRUCTION STAGE } \\
\hline \multicolumn{5}{|c|}{ Sustainability Master Plan Executive Summary (ISCG_SM_ES1, February 2.010) } \\
\hline \multirow{2}{*}{ Target } & \multicolumn{2}{|c|}{ IICG } & \multirow{2}{*}{$\frac{\text { LSC-Jerez }}{(\mathbf{2 . 0 1 1 - 2 . 0 1 2 )}}$} & \multirow{2}{*}{ Rated } \\
\hline & 2011 & 2020 & & \\
\hline$\%$ Local materials & & $40 \%$ & $93 \%$ & $\checkmark$ \\
\hline$\%$ Recycled content & & $40 \%$ & $3 \%$ & $\checkmark$ \\
\hline Tonnes of waste / 100 m2 GLA & 8 & 4,5 & 5,8 & $\checkmark$ \\
\hline$\%$ Waste Recycling or Reuse & $50 \%$ & $75 \%$ & $100 \%$ & $\checkmark$ \\
\hline \% Local labor & $30 \%$ & $50 \%$ & $16 \%$ & $x$ \\
\hline$\%$ Eficient transport & $15 \%$ & $25 \%$ & $90 \%$ & $\checkmark$ \\
\hline
\end{tabular}

1,25

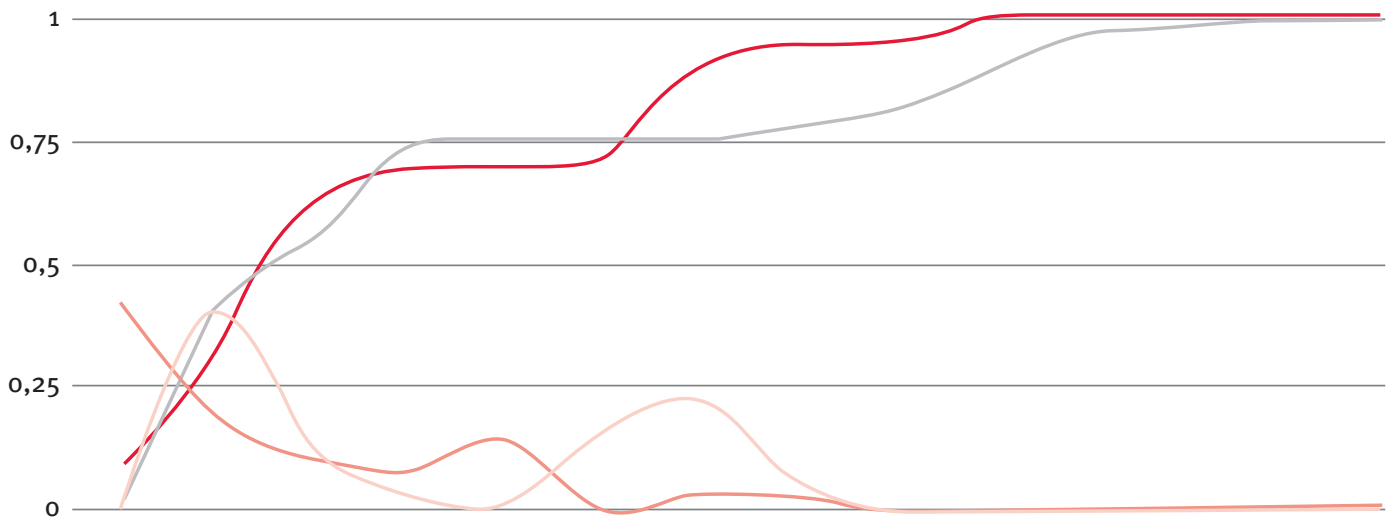

Figure 4.

Sustainability

Targets -

Practice

Case: Retail

park

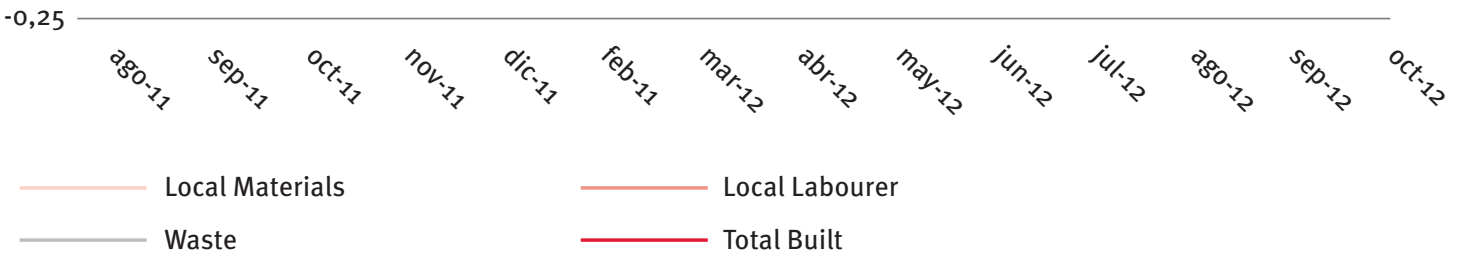




\begin{tabular}{|c|c|c|c|c|}
\hline \multicolumn{5}{|c|}{ Escenarios de Riesgo según el número de trabajadores: } \\
\hline (ud: repeticiones) & Leve & Moderado & Grave & Muy Grave \\
\hline 10-50 Trabajadores & 2 & 0 & 0 & 0 \\
\hline 10-50 Trabajadores & 5 & 0 & 0 & 0 \\
\hline 50-100 Trabajadores & 5 & 1 & 0 & 0 \\
\hline 100-200 Trabajadores & 10 & 1 & 0 & 0 \\
\hline 200-300 Trabajadores & 20 & 1 & 0 & 0 \\
\hline 300-400 Trabajadores & 30 & 1 & 0 & 0 \\
\hline 400-500 Trabajadores & 40 & 1 & 0 & 0 \\
\hline
\end{tabular}

\begin{tabular}{|c|c|}
\hline Contratista & No de trabajadores \\
\hline Aisproyec, S.L. & 16 \\
\hline Cicop, S.A. & 4 \\
\hline CEP, S.L. & 26 \\
\hline Inkema Sistemas, S.L. & 1 \\
\hline Santiga Sistemes, S.L. & 3 \\
\hline Solei Building, S.L. & 6 \\
\hline Terratest, S.A. & 0 \\
\hline TOTAL (A) & 56 \\
\hline
\end{tabular}

\begin{tabular}{|c|c|c|c|c|}
\hline \multicolumn{5}{|c|}{ Valoración de incidencias según Escenario: } \\
\hline (ud: valor en puntos) & Leve & Moderado & Grave & Muy Grave \\
\hline 10-50 Trabajadores & 2 & 5 & 15 & 30 \\
\hline 10-50 Trabajadores & 2 & 5 & 15 & 30 \\
\hline 50-100 Trabajadores & 2 & 5 & 20 & 40 \\
\hline 100-200 Trabajadores & 2 & 5 & 30 & 60 \\
\hline 200-300 Trabajadores & 2 & 5 & 50 & 100 \\
\hline 300-400 Trabajadores & 2 & 5 & 70 & 140 \\
\hline 400-500 Trabajadores & 2 & 5 & 90 & 180 \\
\hline
\end{tabular}

ISI índice semanal incidencias: (B/A) $\quad 0,07$

\begin{tabular}{|c|c|c|c|}
\hline \multicolumn{4}{|c|}{ Valoración de incidencias: } \\
\hline Tipo de incidencias & Valor & Repeticiones & Total \\
\hline Leve & 2 & 2 & 4 \\
\hline Moderada & 5 & 0 & 0 \\
\hline Grave & 20 & 0 & 0 \\
\hline Muy Grave & 30 & 0 & 0 \\
\hline \multicolumn{2}{|c|}{ Valoración de incidencias (B) } \\
Riesgo (C) según escenario
\end{tabular}

Weekly numbers of workers, accidents

scale and risk ratio

and subchapters.

From these criteria defined for real estate projects, the next step of the methodology is to identify the key performance Indicators (KPI) representative for each criteria.

\section{Stakeholders}

Stakeholders are defined as people or groups who are interested in the performance or success of a project, or mandatory to the project (NCB, ver. 3.1)- All of them are capable of direct or indirect influence on the project, not

\section{Figure 5. Health \& Safety criteria - Practice Case: Retail park}

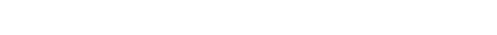




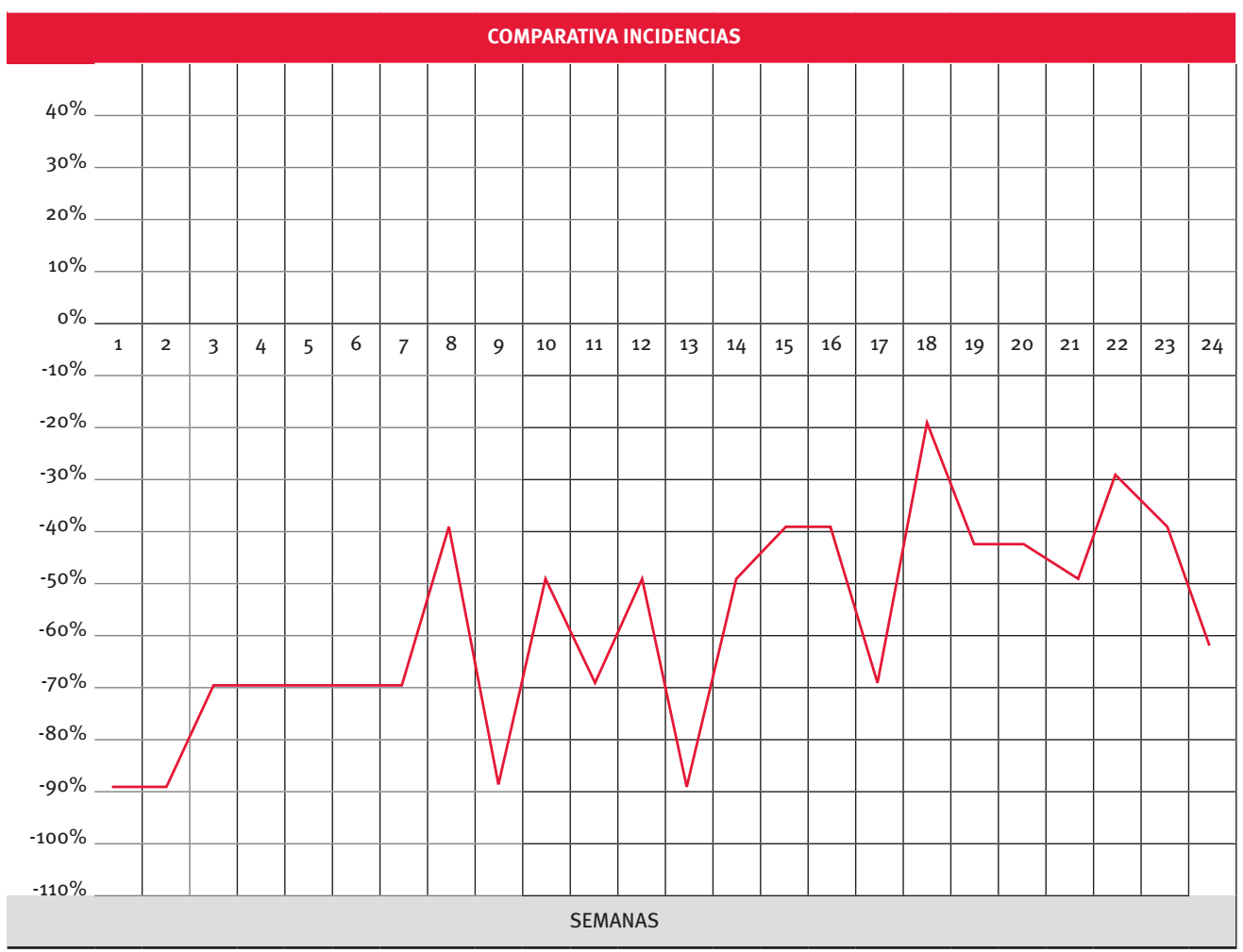

Weekly evolution of health \& safety risks

continuously altering the project management's decision scenarios. As a consequence it is essential for a management plan that includes a methodology for monitoring and measuring the influence of each stakeholder involved within the project's objectives, for all the areas of responsibility defined above.

Making an analysis of the stakeholders of a construction project in Spain, has been found that many of them are common throughout most European countries. The following figure 6 , identifies the stakeholders involved within a Spain's Real Estate Project:

Identifying the Property or Project Promoter to include all the various departments, the sponsor or organization responsible for funding the project, the PMO / Project Management, promoters competitors, not forgetting all stakeholders whom invariably can influence the project's outcome. Legal
Advisers, Planning, law and regulations, public authorities, supply companies -usually private companies indirectly linked to public authorities- stakeholders within the project's design and technical development, stakeholders relative to the construction process, from technical teams, audit, commissioning right through to the contractors. Insurance, including companies and technical control agencies to ensure the project's legal coverage, organizations such as professional associations, Land Registry, Public Notaries and Marketing and Communication. Finally, we mustn't overlook the stakeholders operating phase.

As a result of this analysis it is necessary to implement a Project / portfolio /program methodology for monitoring and measuring the stakeholders influence within the Promoter's areas of responsibilities. The ultimate
Figure 5. Health \& Safety criteria Practice Case: Retail park

goal being to improve the decisionmaking processes by the Project Management.

\section{Methodology}

Once the Promoter's configuration has been defined, its organizational structure and areas of responsibility determined and identified stakeholders of any Project / portfolio / program, it's feasible to outline a methodology for data collection, control and monitoring of objectives related to responsibilities.

The methodology is based on the parameterization of all areas of the promoter's responsibilities throughout the project's life cycle, the proviso being that these responsibilities remain active.

Each area of responsibility can be analysed, proposing the parameterization's process allowing us to measure and for its continual monitoring, e.g. figure 7 Sustainability Construction's KPIs. 


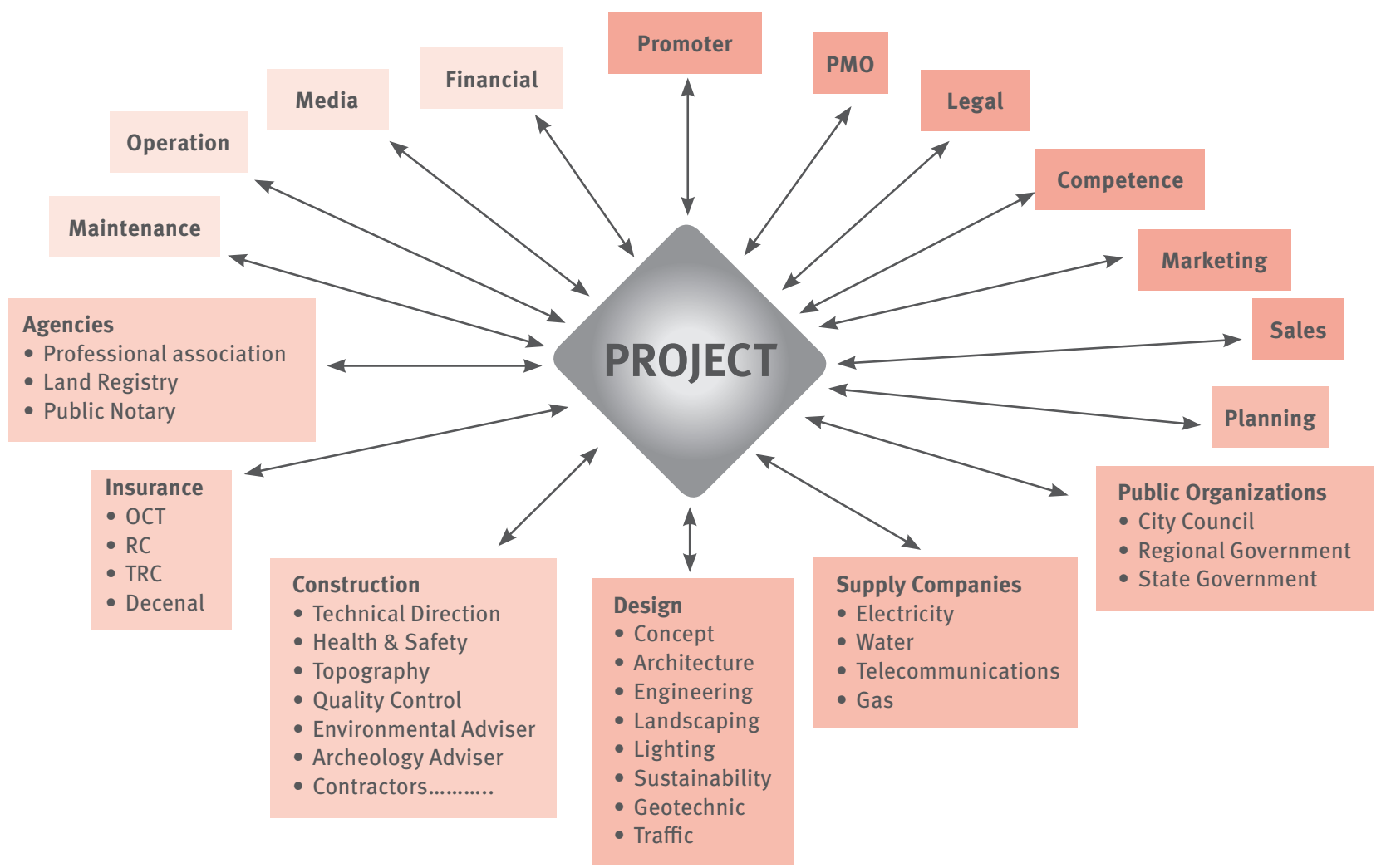

Figure 6. Stakeholders - Spain's real estate project

For each area of responsibility it's feasible to identify the following key performance indicators, showing only primary and secondary data information, in order to simplify the exposition:

\section{Finance:}

Parameterization process:

- Primary Data Secondary Data Key Performance Indicators

- Project / Portfolio / Program Chapter / Subchapter Proposed

- Investment Approval / Investment Approval / Investment split by areas

- Actual Investment /Contracted Investment /Temporary Investment phases

D Deviation Actual I. vs I. Approval /Change Order Investment / Investment by construction chapters

Annual Investment Approval / Investment Commitment

$\checkmark$ Investment Invoices / Investment Invoices
> Investment Invoices / Deviation vs Annual Investment Approval

\section{Planning Time:}

Parameterization process:

- Primary Data / Secondary Data / Key Performance Indicators

> Project / Portfolio / Program Chapter / Subchapter Proposed

- GeneralPlanning Approval / Planning Approval / Ratio of planning by area

> Actual Planning / Actual Planning Ratio of planning by temporary phases

- Actual Planning Deviation vs Planning Approval / Deviation Actual Planning vs Planning Approval / Ratio of planning by construction chapter / subchapter

\section{Production:}

Parameterization process:

- Primary Data / Secondary Data Key Performance Indicators

Project / Portfolio / Program
Chapter / Subchapter Proposed

GeneralProductionApproval / Production Approval /Construction Stage Trend Curves

- Actual Production / Actual Production / Project Construction Trend Curves

- Actual Production Deviation vs Production Approval / Stage Production Ratio

\section{Legal:}

Parameterization process:

- Primary Data / Secondary Data / Key Performance Indicators

- Project / Portfolio / Program Chapter / Subchapter Proposed

- Contracted average total / Contracted average total / Legalities production Trend Curve

- Deactivation average total / Deactivation average total / Legalities Project trend curve

- Theoretical Legalities - average / 
Theoretical Legalities - average

- Actual Legalities -average /

Actual Legalities - average

Quality Control:

Parameterization process:

- Primary Data / Secondary Data Key Performance Indicators

- Project / Portfolio / Program Chapter / Subchapter Proposed

- Actual Test Productivity - average Actual Test Productivity - average / Ratio of negative test by construction stages

- Negative Test Productivity - average / Negative Test Productivity - average / Ratio of negative test by project

> Negative Test Productivity / Deviation vs Actual Test / Productivity / Negative Test Productivity Deviation vs Actual Test Productivity

\section{Sustainability:}

Parameterization process:

> Primary Data / Secondary Data Key Performance Indicators

- Project / Portfolio / Program Chapter / Subchapter / Proposed

$>$ Local Materials - Average / Local Materials - Average / Tonnes of waste per $100 \mathrm{~m} 2$ build

- Recycled content average / Recycled content - average / Average of recycled waste or re-used

Local Labour - average / Local Labour - average

- Efficient transport - average Efficient transport - average

\section{Health \& Safety:}

Parameterization process:

- Primary Data /Secondary Data Key Performance Indicators

- Project / Portfolio / Program Chapter / Subchapter Proposed

- Number of workers / Number of workers per company / Index of weekly risk

- Number of incidences and the accident scale / Number of incidences and accident scale per company

Risk trend curve

\section{Operating / Sales}

Parameterization process:

- Primary Data / Secondary Data Key Performance Indicators

> Project / Portfolio / Program Chapter / Subchapter / Proposed

- Investment Approval / Investment Approval / Investment Stages

> Annuallnvestment Invoices / Contracted Investment Due Diligence productivity - average

- Annual Investment Invocies Deviation vs Investment Approval Change Order Investment

> Due Diligence : Data Room / Investment Commitment

$>$ Investment Invoices

Due Diligence : Data Room

Insurance:

Parameterization process:

- Primary Data / Secondary Data / Key Performance Indicators

> Project / Portfolio / Program Chapter / Subchapter Proposed

$\checkmark$ Risk analysis and Follow up / Risk analysis and Follow up / Number of incidences

Insurance Contract

\section{Maintenance:}

Parameterization process:

- Primary Data / Secondary Data Key Performance Indicators

- Project / Portfolio / Program Chapter / Subchapter Proposed

- Preventive maintenance: completion task-average Preventive maintenance: completion task - average Number of incidences

- Corrective maintenance: completion task-average Corrective maintenance: completion task - average Average time of response

$\checkmark$ Annual Approval Maintenance Investment / Preventive Maintenance Investment vs Total Maintenance Investment

Maintenance Investment Invoices
Finally all the information described above is summarized in simple dashboards, organised either by the Project /program /portfolio, e.g. figure 8 shows a construction subchapter's dashboard.

Data collection is continuous throughout the project, obtaining realtime information and allowing for relevant decision-making based on accurate, realistic and factual knowledge.

\section{Practice Case}

Project: Luz Shopping - Retail Park (Figure 9)

Location: Jerez de La Frontera - Spain

Plot Area: $561.845 \mathrm{~m} 2$

Built Area: $115.972 \mathrm{~m} 2$

Project: Food Logistic warehouse (Figure 10)

Location: Murcia - Spain

Plot Area: $148.101 \mathrm{~m} 2$

Built Area: $67.925 \mathrm{m2}$

\section{Conclusions and future developments}

The continuous parameterization of projects / portfolios / programs, monitoring in real-time and its outcomes using KPI's throughout the project's life cycle, thus allowing the Promoter to obtain the Projects/portfolios/programs full know-how. From this data, the Promoter can transform it into comprehensive information and knowledge, permitting comparisons between more than one project in all areas of responsibility. The Project Portfolio Management can be used by the construction Promoter. Figure 11, shows Retail Portfolio Projects Construction Stages Production - trend curves.

Parameterization methodology enables medium to large sized portfolios/ programs (i.e. more than ten projects) correlations between KPI's from differing areas of responsibility. This feature offers the construction Promoter potential for future development, thus allowing anticipation of all risk scenarios, i.e. checking 


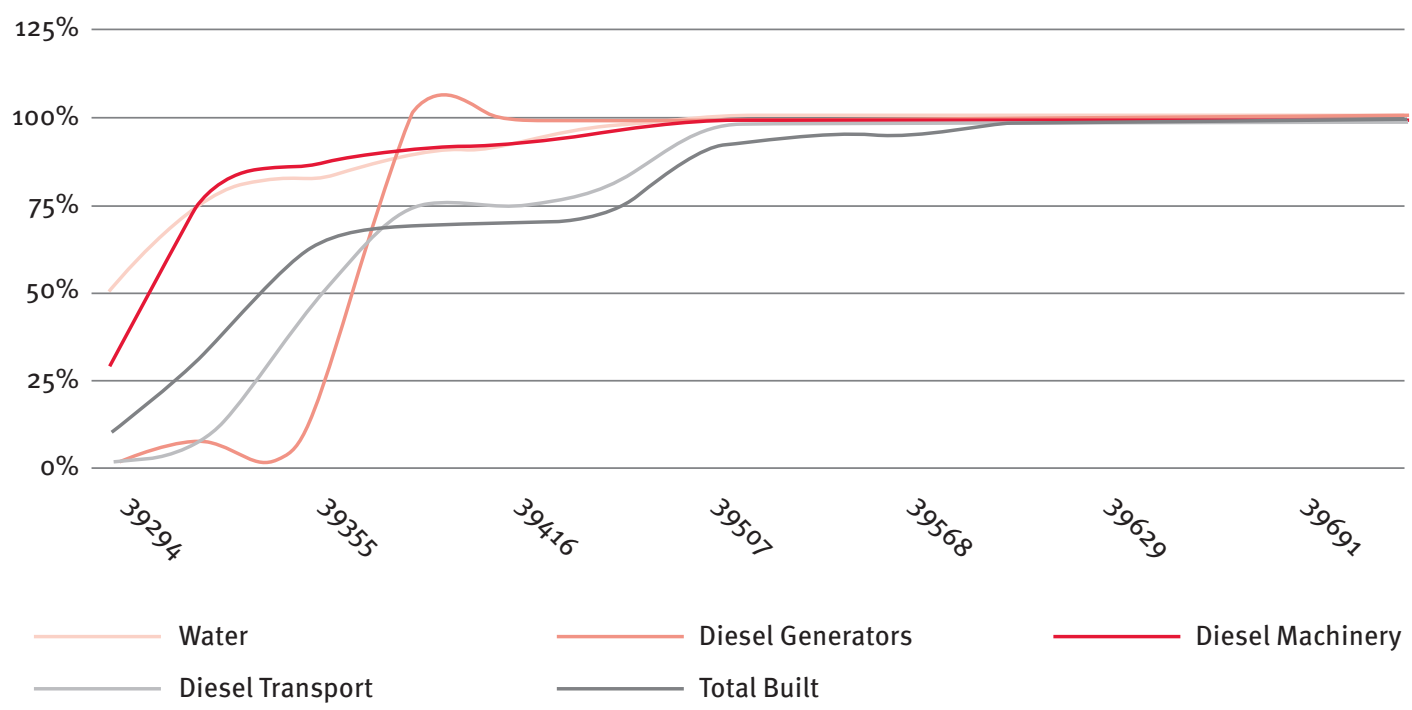

KPI-02

$125 \%$

$100 \%$

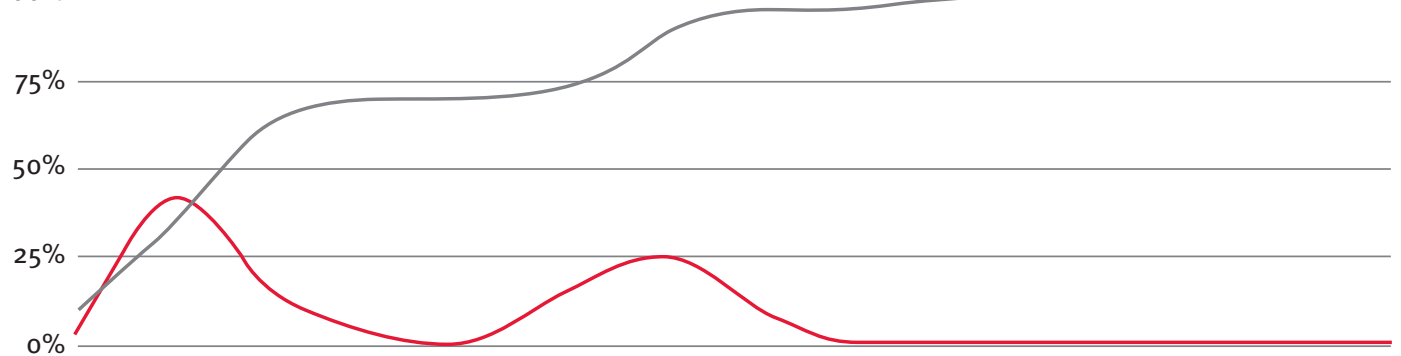

$-25 \%$

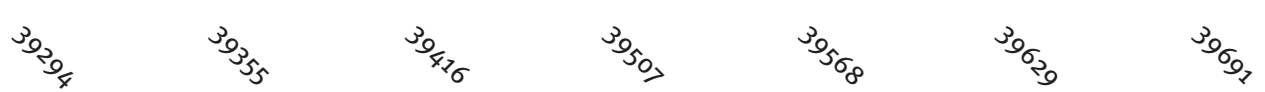

Local Materials

Total Built

Figure 7. Sustainability Construction's KPIs Evolution in time -

Practice Case: Retail park 
KPI-03

$125 \%$

$100 \%$

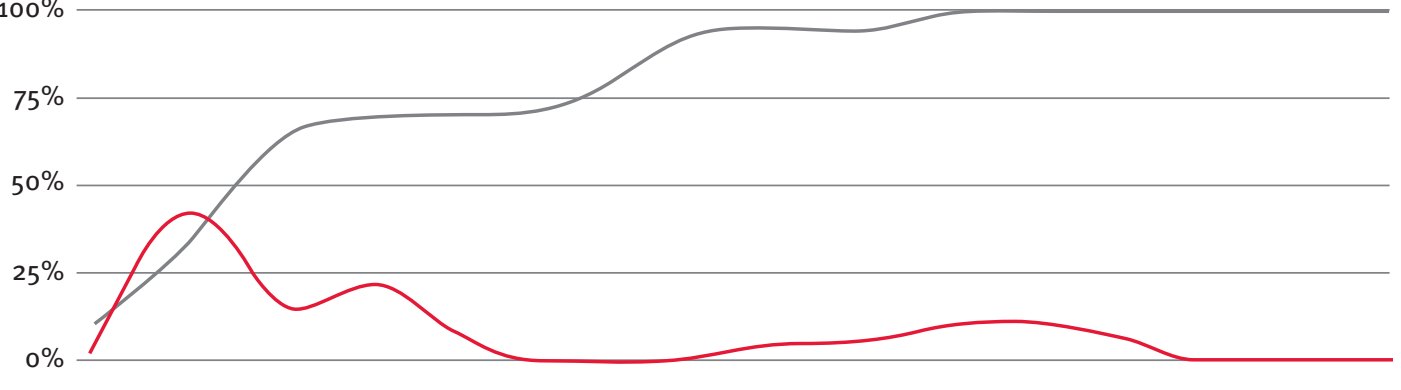

$-25 \%$

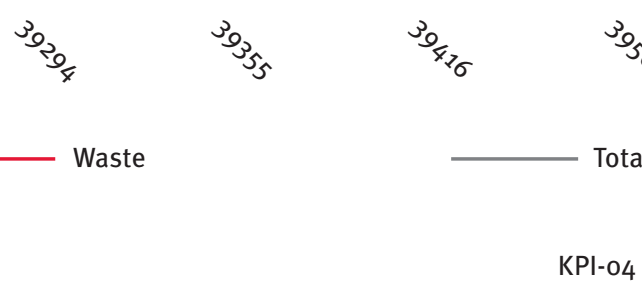

$125 \%$

$100 \%$

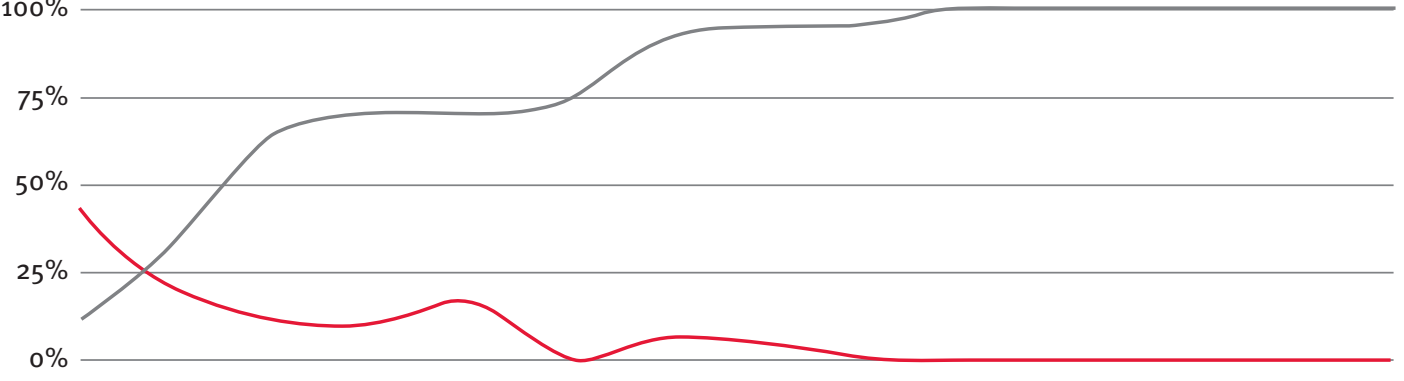

$-25 \%$

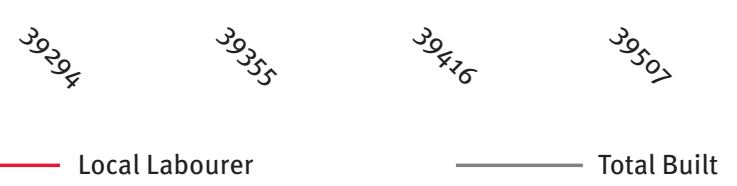

1238 ORGANIZATION, TECHNOLOGY AND MANAGEMENT IN CONSTRUCTION - AN INTERNATIONAL JOURNAL · $7(1) 2015$ 


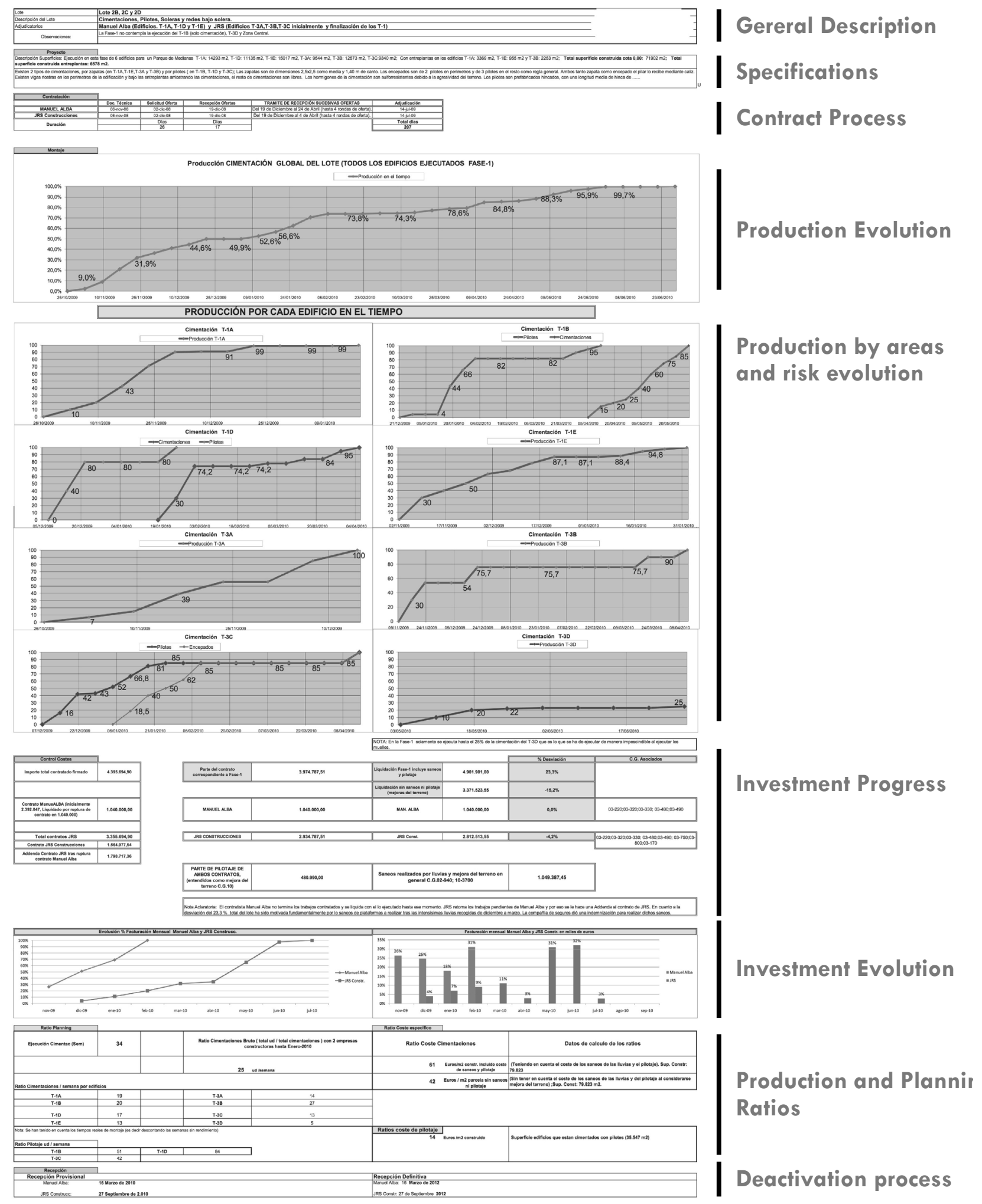

Figure 8. Construction Subchapter's Dashboard - Practice Case: Foundations, Retail park 


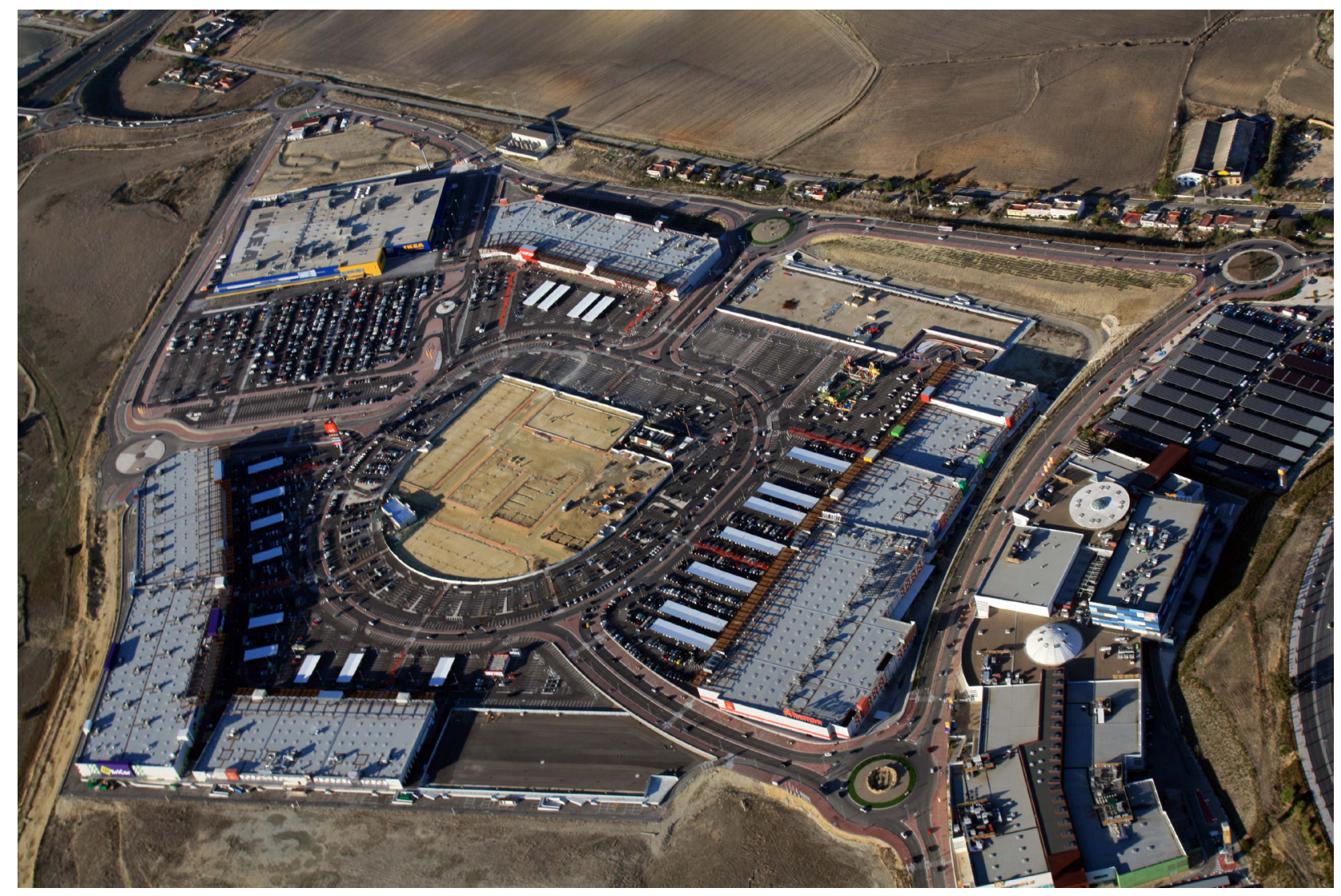

Figure 9. Practice Case Luz Shopping Retail park, Jerez de La Frontera, Spain

the real project's figures against the model's figures attained using this methodology.

Presently, 10t Project Management is transferring this parameterization responsibilities' methodology created for the construction promoters use to a information and communications technology (ICT) tool, thus permitting the promoter's systematic implementation of their projects / portfolio / programs. This methodology will assist promoters in developing their business inteligence.

\section{References}

Heredia, R. (1999), Dirección Integrada de Proyecto - DIP- Project Management, Alianza Editorial, Madrid (Spain).

Adams and McCaffer (1999), Modern Construction Management, Blackwell Science Ltd, Oxford.

Domingo, A. (2000), Dirección y Gestión de Proyectos Un enfoque práctico, Ra-Ma editorial, Madrid (Spain).

Gómez, J. (2000), Gestión de Proyectos, Fundación Confemetal Editorial, Madrid.

Martínez, F. (2001), Formularios de contratos para constructores y promotores, CISSPRAXIS, Valencia (Spain).
Martínez, J (2006), NCB Bases para la competencia en Dirección de Proyectos versión 3.1, Editorial UPV, Valencia (Spain).

Turner, R. (2009), The Handbook of ProjectBased Management, Leading Strategic Chance in organization, McGraw Hill.

López Paredes, A. (2013), Certificación IPMA4LC, bpmsat, Valladolid (Spain).

Díez, S. (2015), La Transparencia que demanda el sector inmobiliario se llama Sofia RTD, Observatorio Inmobiliario, no -73 , pp 52-53.

Díez, S. (2015), Las diez responsabilidades de todo promotor inmobiliario, available at: https://www.sofia-rtd.com/?p=2441 (accessed 30 april 2015). 


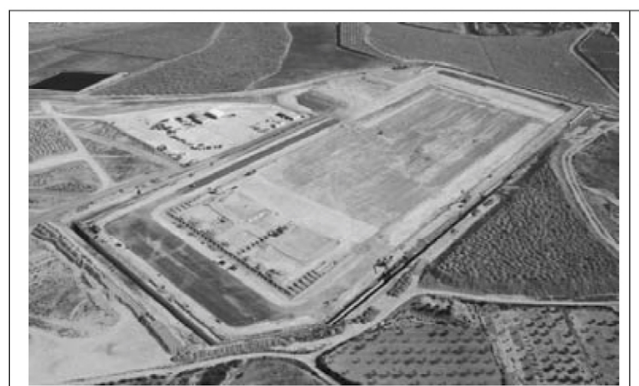

FEBRERO 2011

$5 \%$

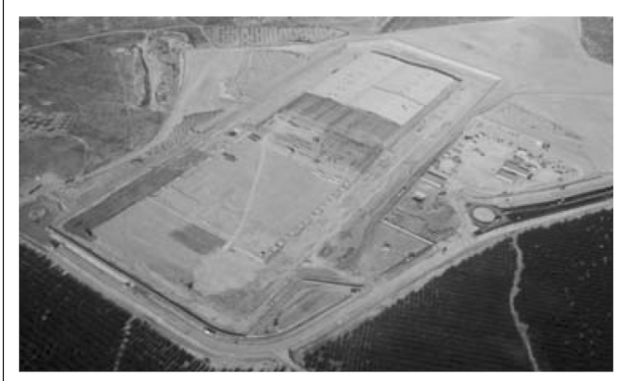

JUNIO 2011.

$20 \%$

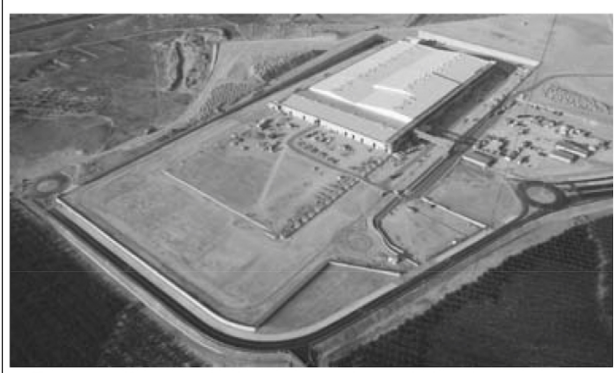

OCTUBRE 2011

$42 \%$

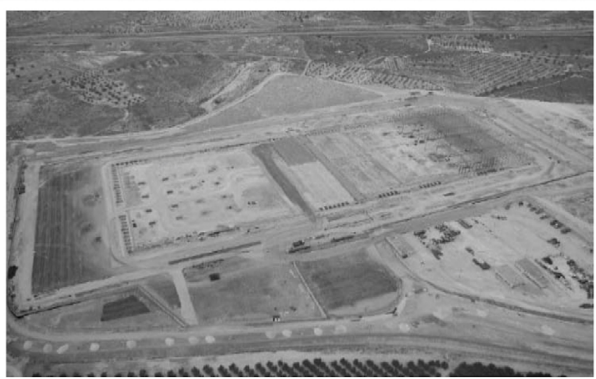

ABRIL 2011

$10 \%$

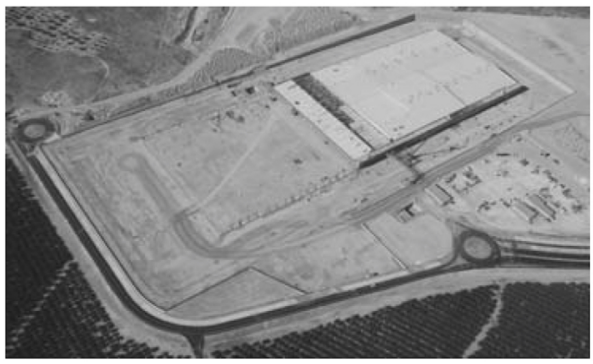

AGOSTO 2011.

$33 \%$

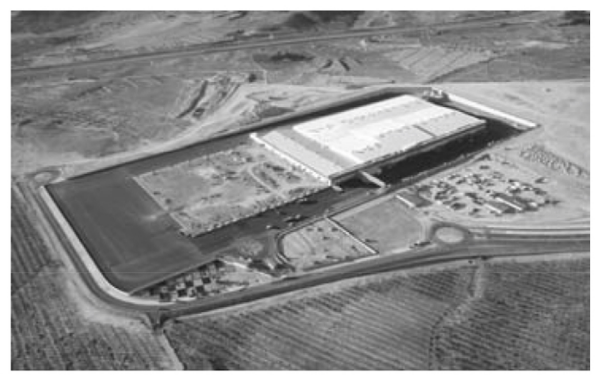

DICIEMBRE 2011

$67 \%$

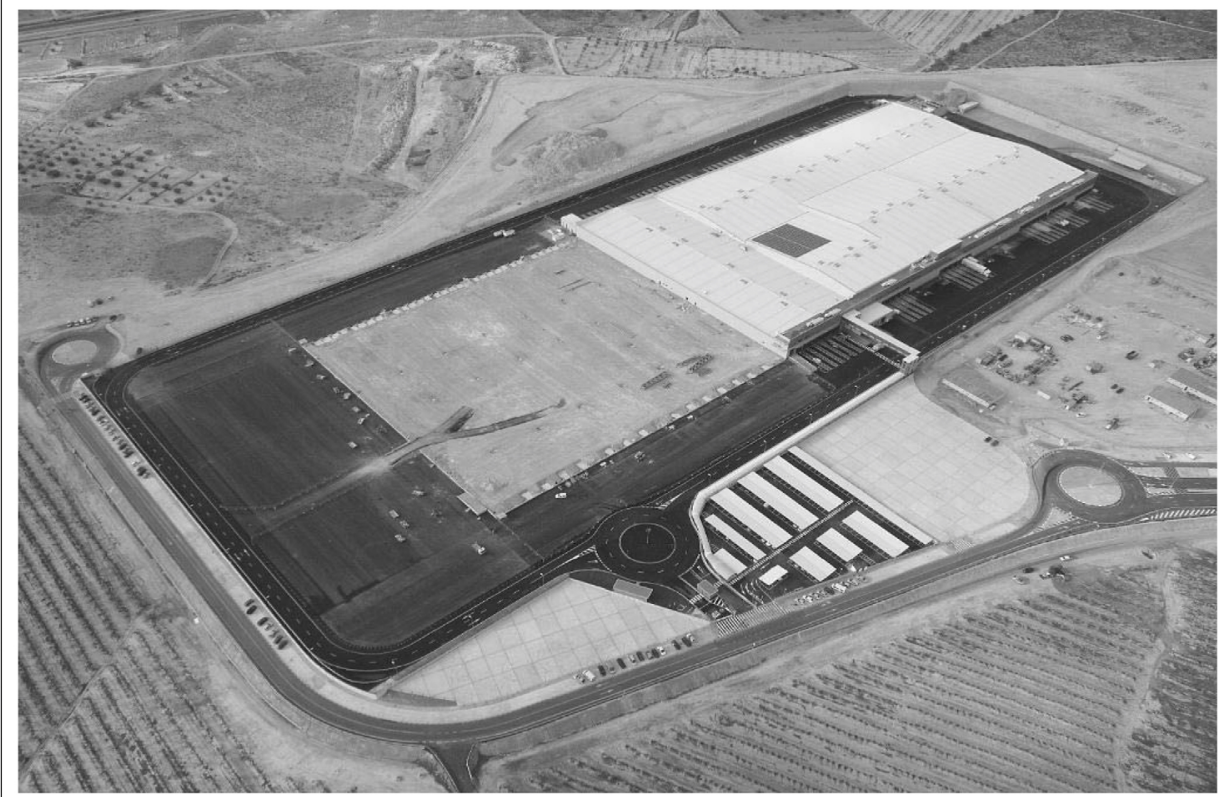

FEBRERO 2012.

$79 \%$ FIN FASE I 


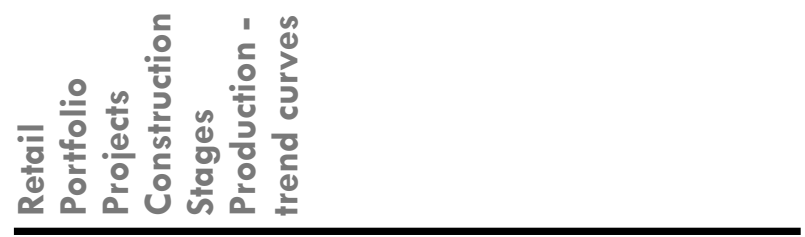
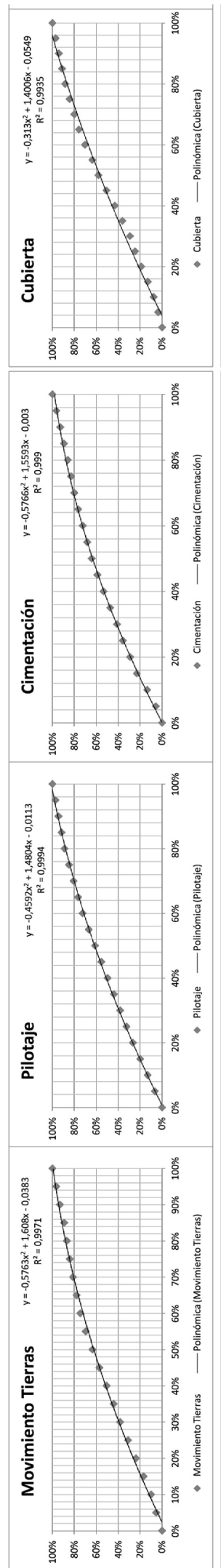
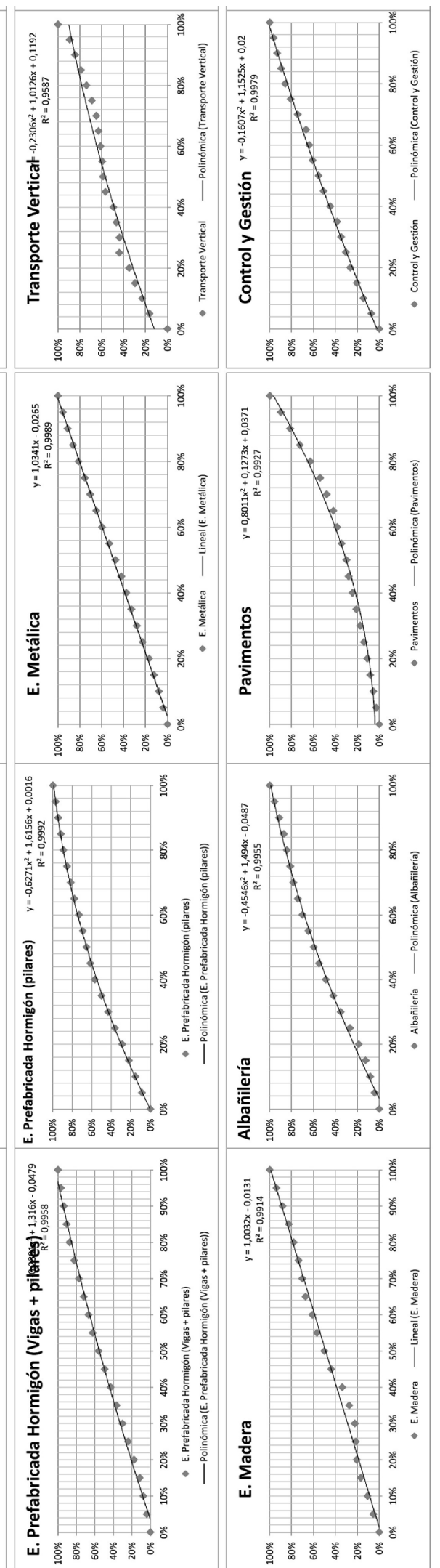

Figure 11: Construction

Subchapter's Trend Curve:

foundations, concrete

structure precast, wood structure, steel structure, masonry, flooring. 